\title{
Simplified treatment of the radiative transfer problem in expanding envelopes
}

\author{
J. Gómez-Gomar ${ }^{1}$ \\ and \\ J. Isern ${ }^{1}$
}

Received —

Submitted to: ApJVersion: December 23, 2017

\footnotetext{
${ }^{1}$ Centre d'Estudis Avançats de Blanes (CSIC), Blanes, Camí de Santa Barbara s/n, C.P. 17300, Girona, Spain.
} 


\begin{abstract}
In this paper we study the application of a simplified method to solve the dynamic radiative transfer problem in expanding envelopes. The method, which requires a computational effort similar to that of the diffusion approximation, is based on the use of a generalization of the Eddington closure relationship allowing the inclusion of scattering and relativistic corrections to $O(v / c)$. We apply this method to the calculation of light curves of type Ia supernovae, showing that it gives much more accurate results than the diffusion approximation, and that the latter is seriously in error when applied to determine emergent flux and its spectral distribution.
\end{abstract}

Subject headings: radiative transfer - supernovae: general 


\section{Introduction}

Radiation transfer plays a very important role in many astrophysical systems. In the case of expanding extended envelopes, like those found on supernova or nova outbursts, this

problem turns out to be crucial since the optically thin region represents a non-negligible part of the system. The evolution of these envelopes is strongly influenced, or even dominated, by radiation and their detailed modeling is complex and time consuming.

Very often the "diffusion approximation" is used to study these problems. Arnett 1979, Arnett 1980, Kato et al. 1994). However, the basic hypotheses lying beneath this method, which is accurate in optically thick media, are no longer valid in the envelopes considered here. First of all, the mean free path of the photons and the characteristic length of the envelope are of the same order. As a consequence, the radiation field becomes anisotropic due to the geometrical effects. Secondly, the energy distribution of the radiation is not coupled to the local properties of matter, specially if scattering is dominant, and the contribution of non-local radiation to the continuum makes inadequate the assumption of thermal equilibrium between matter and radiation.

To circumvent this problem the general moment equations (from which the diffusion approximation is derived) is used when accurate treatments are required. However, to solve the moment equations it is necessary to add a closure equation to obtain a unique solution. This "closure relationship" is not known "a priori", except in some particular cases like those where the well known Eddington relationship $\left(P_{\nu} / E_{\nu}=1 / 3\right)$ holds. Very often the "variable Eddington factor" is used. In this case, the closure relationship is obtained by solving, from time to time, the complete transfer equation in its static version. The radiation moments are then computed from the intensities determined in this way and the variable Eddington factor $\left(f_{\nu}=P_{\nu} / E_{\nu}\right)$ is obtained (Stone et al. 1992, Höflich et al.1993). Although this method is quite efficient, it still demands an important computational effort 
in highly dynamical systems, where the transfer equation must be solved frequently.

A generalization of the Eddington relationship valid for static extended envelopes was developed by Simonneau (1979). The efficiency of this approximation was tested in static envelopes similar to those found in supernova outbursts (except for the velocity field ) in the cases where scattering was absent or dominant (Lopez, Simonneau \& Isern 1987, Simonneau, Isern \& Lopez 1989). The main advantage of this relationship is that it can be easily obtained a priori. In this paper we test the use of this relationship in the dynamic case and we show that, although the computational requirements of this method are similar to those of the the diffusion approximation, the results are noticeably improved.

\section{The model}

As the test scenario we have chosen the semi-analytic model for a SNIa proposed by Schurmann (1983). The expanding structure is composed by a $0.8 \mathrm{M}_{\odot}$ constant density core, surrounded by a $0.6 \mathrm{M}_{\odot}$ envelope with a density profile $\rho \propto r^{-7}$. The object is homologously expanding with a total kinetic energy of $1.2 \times 10^{51}$ ergs. The core contains 0.7 $\mathrm{M}_{\odot}$ of totally burned matter $\left(\approx 100 \%{ }^{56} \mathrm{Ni}\right.$ just after the explosion $)$, and a $0.1 \mathrm{M}_{\odot}$ partially

burned mantle, while the envelope is formed by unburned $\mathrm{C} / \mathrm{O}$. The only source of energy is the radioactive decay of ${ }^{56} \mathrm{Ni}$ and ${ }^{56} \mathrm{Co}$ in the core. The energy deposited by positrons and $\gamma$-rays produced in the decays is given by an analytic fit to Monte Carlo simulations performed by Colgate and Petschek (1980) for very similar models.

Three different total opacities have been used: $\chi=0.05 \rho \mathrm{cm}^{-1}, \chi=0.2 \rho \mathrm{cm}^{-1}$ and pure free electron opacity (temperature and density dependent). In all the cases, the opacities are assumed to be independent of the frequency. The temperature dependent opacity presents a rather realistic evolution but, since no line contributions were considered, 
it might fall to too low values at late phases. To avoid that, a lowest value of $\chi=0.005 \rho$ $\mathrm{cm}^{-1}$ was imposed. Different parameterizations were also adopted for the fraction of pure absorption: $\epsilon=1,0.1$ and 0.01 .

Since we are interested in highly dynamic systems the effects of velocity on the radiation field have to be considered (Castor 1972, Mihalas et al. 1978). If all the relativistic terms to $O(v / c)$ are included and the presence of scattering is considered, the comoving frame transfer equation takes the form (Mihalas \& Weibel-Mihalas 1984):

$$
\begin{array}{r}
\frac{1}{c} \frac{\partial I_{\nu}(r, \mu, t)}{\partial t}+\beta \frac{\partial I_{\nu}(r, \mu, t)}{\partial r} \\
+\frac{\mu}{r^{2}} \frac{\partial\left(r^{2} I_{\nu}(r, \mu, t)\right)}{\partial r}+ \\
\frac{\partial}{\partial \mu}\left[\left(1-\mu^{2}\right)\left(\frac{1}{r}+\mu\left(\frac{\beta}{r}-\frac{\partial \beta}{\partial r}\right)\right) I_{\nu}(r, \mu, t)\right] \\
-\frac{\partial}{\partial \nu}\left[\nu\left(\left(1-\mu^{2}\right) \frac{\beta}{r}+\mu^{2} \frac{\partial \beta}{\partial r}\right) I_{\nu}(r, \mu, t)\right] \\
+\left(\left(3-\mu^{2}\right) \frac{\beta}{r}+\left(1+\mu^{2}\right) \frac{\partial \beta}{\partial r}\right) I_{\nu}(r, \mu, t) \\
=\chi_{\nu}\left(\epsilon_{\nu} B_{\nu}(T)-\left(1-\epsilon_{\nu}\right) J_{\nu}\right)-\chi_{\nu} I_{\nu}(r, \mu, t)
\end{array}
$$

with,

$$
\chi_{\nu}=\sigma_{\nu}+\kappa_{\nu}
$$

and,

$$
\epsilon_{\nu}=\frac{\kappa_{\nu}}{\chi_{\nu}}
$$

$\sigma_{\nu}$ and $\kappa_{\nu}$ are the scattering and pure absorption components of the opacity and $\epsilon_{\nu}$ is defined as the pure absorption fraction. The other quantities have their usual meaning. The scattering is assumed to be coherent and isotropic and its presence makes the equation (四) to become integro-differential due to the term $J_{\nu}$.

Integrating (四) over frequency and calculating its first and second moments respect to 
$\mu$ it is possible to obtain the first and second frequency integrated moment equations.

$$
\begin{aligned}
& \frac{1}{c} \frac{\partial J}{\partial t}+\beta \frac{\partial J}{\partial r}+\frac{1}{r^{2}} \frac{\partial\left(r^{2} H\right)}{\partial r}+\frac{\beta}{r}(3 J-K)+\frac{\partial \beta}{\partial r}(J+K) \\
& =\left(\chi_{\mathrm{P}}-\sigma_{\mathrm{P}}\right) B(T)-\chi_{\mathrm{J}} J+\sigma_{\mathrm{J}} J \\
& \frac{1}{c} \frac{\partial H}{\partial t}+\beta \frac{\partial H}{\partial r}+\frac{\partial K}{\partial r}+\frac{3 K-J}{r}+2\left(\frac{\partial \beta}{\partial r}+\frac{\beta}{r}\right) H \\
& =-\chi_{\mathrm{H}} H
\end{aligned}
$$

In (4) and (5) the "mathematical" radiation moments are related to the "physical" ones just by $E=\frac{4 \pi}{\mathrm{c}} J, F=4 \pi H$ and $P=\frac{4 \pi}{\mathrm{c}} K$. Since the equations are frequency integrated, the opacities $\left(\chi_{H}, \chi_{J}\right.$ and $\left.\chi_{P}\right)$ are mean opacities: the flux mean, the absorption mean and the Planck mean respectively. In this work only grey opacities are considered and thus $\chi_{H}=\chi_{J}=\chi_{P}$.

Since the general moment equations (4) and (5)contain three unknowns it is necessary to add a third equation:

$$
\Phi(J, H, K, r, t)=0
$$

This equation is known as the closure relationship and it recovers part of the geometrical information lost after integrating (4) over $\mu$. Notice that the set formed by the equations (田), (5) and (6) is equivalent to the diffusion approximation in the asymptotic limit for optically thick media:

$$
\begin{gathered}
\frac{1}{c} \frac{\partial J}{\partial t}+\beta \frac{\partial J}{\partial r}+\frac{1}{r^{2}} \frac{\partial\left(r^{2} H\right)}{\partial r}+\frac{\partial \beta}{\partial r} \frac{4}{3} J=\kappa_{\mathrm{P}}(B(T)-J) \\
\chi_{\mathrm{R}} H=-\frac{1}{3} \frac{\partial J}{\partial r}
\end{gathered}
$$


Although the closure relationship has apparently disappeared, it is implicitly included. Furthermore, several velocity dependent terms as well as the scattering contribution have been dropped and the flux is instantaneously determined by the energy density distribution since no time derivative appears in equation (8). All these properties simplify the numerical treatment of the problem, but its accuracy decreases as the optical depth of the medium decreases.

A remark valid for both sets of equations is that all the radiation moments appearing in them are comoving moments. For this reason they always have to be transformed to inertial frame moments to compare with observations. This is done by using the transformation equations (Mihalas \& Weibel-Mihalas 1984):

$$
\begin{gathered}
J^{\prime}=J+2 \beta(H) \\
H^{\prime}=H+\beta(J+K) \\
K^{\prime}=K+2 \beta(H)
\end{gathered}
$$

There are two cases where (6) is known a priori: planar media with grey opacity and optically thick regions, where the radiation field is quasi-isotropic. In the former case an essentially exact, in the latter the particularly simple Eddington closure relationship (12) holds.

$$
3 K-J=0
$$

When the anisotropies of the radiation field due to geometrical effects become important, the equation (12) is no longer valid. An example is the radiation field far away from a quasi-punctual source which verifies $K-J \approx 0$ (Stone et al. 1992). Nevertheless, due to its simplicity, equation (12) is very often applied and, in fact, it is assumed when using the diffusion approximation. 
Generally, when (12) fails, the "variable Eddington factor" $(K / J=P / E=f(r, t))$ is used. The factor is computed solving from time to time the stationary transfer equation and it is assumed to be constant between successive determinations. This method implies an important increase in the complexity and time consumption of the calculations.

Simonneau (1979) proposed a generalization of equation (12) for spherically symmetric systems where radiation anisotropies are important. In the grey case, the equation takes the form:

$$
3 K-J=2 \mu_{c} H \quad\left(0 \leq \mu_{c} \leq 1\right)
$$

where the parameter $\mu(r, t)_{c}$ is given by:

$$
\frac{d \mu_{c}}{d r}=\frac{1-\mu_{c}}{r \mu_{c}} \mathrm{e}^{-\frac{\tau}{\mu_{\mathrm{c}}}} \quad \mu_{\mathrm{c}}(0)=0
$$

It reproduces the equation (12) $\left(\mu_{c}=0\right)$ and the streaming limit $\left(\mu_{c}=1\right)$ as extreme cases. In previous papers (Lopez et al. 1987, Simonneau et al. 1989) it was proven the efficiency of (14) and (13) to produce a suitable closure relationship for static and stationary envelopes.

However, high velocities produce aberration and Doppler shift effects on the travelling photons and they might modify the form of this closure relationship respect to the the static case. With the purpose of evaluating such effects, several stationary but non-static envelopes with suitable velocity fields have been examined. The radiation field of these systems was determined by solving the complete transfer equation with and without a velocity field. In the former case the moments were obtained, both in the comoving-frame and the inertial-frame. Values of $\mu_{c}$ were computed using (13). Figure (11) displays the profiles obtained for one of these envelopes. In all the cases the three profiles were similar. In particular, the differences between the static value and the non-static one in the inertial frame are negligible. The conclusion is that the closure relationship for the inertial-frame moments of the radiation field was not noticeably affected by the presence of velocity fields (at least, in the moderately-relativistic case). The reason for that is that the values adopted 
by $\mu_{c}$ are only significant (i.e. $\mu_{c} \nless 1$ ) in low opacity regions, where the radiation is not strongly coupled to the local conditions and velocities do not have a significant influence on the photon distribution.

\section{EDITOR: PLACE FIGURE 1 HERE.}

Once it is assumed the invariance of the closure relationship for the inertial-frame moments, it is possible to write in the comoving-frame:

$$
3 K\left(1+\frac{2}{3} \mu_{c} \beta\right)-J\left(1+2 \mu_{c}\right)=2 \mu_{c} H\left(1+2 \frac{\beta}{\mu_{c}}\right)
$$

Which is the final expression we used in this work.

On the other hand, it is also necessary to estimate the validity of the hypothesis of stationarity on the expression (15). The necessary and sufficient condition for its applicability in dynamic situations is:

$$
\tau_{\text {radiation }} \ll \tau_{\text {evolution }}
$$

where $\tau_{\text {radiation }}$ is the characteristic time for the evolution of the radiation field and $\tau_{\text {evolution }}$ is the characteristic time for the evolution of the system. In regions where the role of (15) is relevant (optically thin), $\tau_{\text {radiation }}$ corresponds to the free flight time (Mihalas \& Weibel-Mihalas 1984), which is generally shorter than the evolution time for any fluid flow system. In particular, in the case of SNIa, $\tau_{\text {evolution }}$ is determined by the dynamical time and the decay time of ${ }^{56} \mathrm{Ni}$ and ${ }^{56} \mathrm{Co}$, which are indeed much longer than $\tau_{\text {radiation }}$. The use of (15) and (14) is then justified.

The calculation of a model of supernova envelope involves not only the integration of the equations describing the radiation field but also those describing the evolution of matter. Fortunately, there are several properties of supernovae that make possible to 
simplify the problem: i) During the homologous expansion, the structure of the envelope can be easily determined if the initial velocity profile is known. ii) Due to the low densities and high temperatures, the energy content of the envelope is dominated by radiation, and since radiative balance of the gas can be assumed, it is possible to replace the internal energy equation by an equilibrium condition. With these assumptions and using the general moment equations, the expressions which describe the evolution of the energy density take the form (now in Lagrangian coordinates):

$$
\begin{gathered}
\frac{1}{c} \frac{\partial J}{\partial t}+4 \pi \rho \frac{\partial\left(r^{2} H\right)}{\partial M}-\beta \frac{(3 K-J)}{r}-\frac{(J+K)}{c} \frac{\partial \rho}{\partial t} \frac{1}{\rho}=\frac{\xi \rho}{4 \pi} \\
4 \pi \epsilon \chi\left(B\left(T_{\text {gas }}\right)-J\right)=\xi \rho
\end{gathered}
$$

Alternatively, if the diffusion approximation is considered (and hence Eq. 7), the corresponding equations will be:

$$
\begin{gathered}
\frac{1}{c} \frac{\partial J}{\partial t}+4 \pi \rho \frac{\partial\left(r^{2} H\right)}{\partial M}-\frac{4}{3} \frac{J}{c} \frac{\partial \rho}{\partial t} \frac{1}{\rho}=\frac{\xi \rho}{4 \pi} \\
T_{\text {gas }}=T_{\text {rad }}
\end{gathered}
$$

where $\xi$ is the radioactive energy deposition function. For simplicity the gas was considered to be in LTE, although a really accurate description of the problem, which is beyond the scope of this work, would require a NLTE modeling (Baron et al. 1996). Hence, the ionization degree was computed with the Saha equations. In fact, in the calculations this quantity was from the tables previously previously obtained by Bravo et al. (1993). The resulting set of differential equations has been solved using an implicit scheme. The finite difference equations were space and time centered and second order accurate. The complexity of both sets of equations (diffusion approximation and moments) is similar and we have found that their time consumption is almost similar.

In order to verify the quality of the different approximations, we compare the results obtained with them with those provided by the variable Eddington factor method which, as 
in other works, is assumed to give correct results (Stone et al. 1992, Blinnikov et al. 1993, Höflich et al.1993, Ensman 1994). In this case in order to compute the variable Eddington factor, the transfer equation is solved periodically in its stationary form with a second order accuracy scheme, following the parallel ray technique (Mihalas \& Weibel-Mihalas 1984).

Besides the evolution of the bolometric quantities: J, H and K, we have also computed the color temperature, $\mathrm{T}_{U B}$, and the $\mathrm{U}, \mathrm{B}$ and $\mathrm{V}$ light curves. To do that, the stationary monochromatic moment equations have been solved at fixed frequencies and times using

the values of $\mathrm{J}$ and $\mathrm{T}_{\text {gas }}$ obtained with the dynamic frequency-integrated equations and introducing a correction factor to make them consistent with the previously computed values of $\mathrm{H}$.

\section{Results and discussion}

The bolometric light curves obtained with the different approximations are displayed in Figure 2 in one of the cases considered here while Table 11 summarizes the main differences among them. In all the cases, the generalized Eddington factor gives excellent results. At the maximum, the light curve is slightly underluminous and the differences are in the range of $0.02^{m}-0.05^{m}$. In contrast the light curve obtained with the diffusion approximation is clearly overluminous near the maximum although these discrepancies disappear a week or two later, when the luminosity balance is reached. These discrepancies range from $-0.14^{m}$ to $-0.2^{m}$, being more important in the case of models with lower optical depths at maximum, although this dependence is not very strong. The errors in this case are not affected by the adopted value of $\epsilon$. The diffusion approximation also modifies the position of the maximum luminosity which occurs 1-2 days too early. See Table 1

EDITOR: PLACE FIGURE 2 HERE. 
Deviations of $\mathrm{E}_{\text {rad }}(r)=4 \pi / c \mathrm{~J}(r)$ and of $\mathrm{T}_{g a s}(r)$ are also very small for the proposed method. During the first 3 months the errors for these quantities in the innermost and outermost layers are negligible. They appear only in the intermediate region, where they fluctuate above and below the correct values (Fig. 3). In this case the deviations do not disappear at late times but grow as the models evolve. During all this period, relative discrepancies are less than $15 \%$ and $4 \%$ for $\mathrm{E}_{\text {rad }}(\mathrm{r})$ and $\mathrm{T}_{g a s}(\mathrm{r})$ respectively.

\section{EDITOR: PLACE TABLE 1 HERE.}

For the same models the discrepancies introduced by the diffusion approximation appear at the surface just after the maximum luminosity and follow the recession of the photosphere towards inner regions. The estimated values for $\mathrm{E}_{\text {rad }}$ and $\mathrm{T}_{\text {gas }}$ are always below the expected ones and their profiles are too smooth. Two months after the explosion $\mathrm{E}_{\text {rad }}$ is on average $\approx 50 \%$ of the correct value and $\mathrm{T}_{\text {gas }} \approx 30 \%$ and the simulated envelopes become quasi-isothermal, in opposition to those obtained with the variable Eddington factor method. When the optical depth of the models is close to 1 the diffusion approximation is unable to produce useful values for these quantities.

\section{EDITOR: PLACE FIGURE 3 HERE.}

\section{EDITOR: PLACE FIGURE 4 HERE.}

The deviations of $\mathrm{E}_{\text {rad }}$ and $\mathrm{T}_{g a s}$ affect the computation of the monochromatic light curves, the color temperature and the $\mathrm{B}-\mathrm{V}$ index. The excessively low values of $\mathrm{E}_{\text {rad }}$ given by the diffusion approximation cause a redshift of the estimated spectra when models become transparent, This produces a decrease of the color temperature and an increase of 
$\mathrm{B}-\mathrm{V} . \mathrm{T}_{U B}$ displays moderate deviations in the region of the maximum luminosity $(-700 \mathrm{~K}-$ $100 \mathrm{~K}$ ) but, when the total optical depth of the models is $<2$ it steeply falls to very low values as it is displayed in Figure 4. Similarly B-V evolves close to the correct behaviour during the first weeks but it takes too high values as soon as $\mathrm{T}_{U B}$ starts decreasing (Fig. 6). The U, B and V light curves are influenced by the combination of the spectral redshift and the overluminosity at maximum caused by the diffusion approximation. Near the maximum of luminosity, the second effect compensates or even dominates the spectral redshift, but a month later, even the model with the highest opacity is underluminous in the $\mathrm{U}, \mathrm{B}$ and $\mathrm{V}$ bands (Figure 5). At 40 days, the deviations range from $0.2^{m}$ to $3^{m}$. All these discrepancies are sensitive not only to the total opacity, but also to the pure absorption fraction. See Table 1

\section{EDITOR: PLACE FIGURE 5 HERE.}

\section{EDITOR: PLACE FIGURE 6 HERE.}

Once again, the accuracy provided by the generalized Eddington factor for these quantities is excellent. Even 3 to 4 months after the explosion $\mathrm{T}_{U B}, \mathrm{~B}-\mathrm{V}$ and the $\mathrm{U}, \mathrm{B}$ and V light curves evolve very close to the "standard" values obtained with the variable Eddington factor method. In all the models the deviations from the standard results are negligible near the maximum of luminosity. Only after two months it is appreciated a moderate shift towards the blue. During the first 4 months $\Delta \mathrm{T}_{U B} \leq 100$ and the B-V color excess is $\approx-0.05^{m}$. For the same interval the absolute errors of the monochromatic light curves are always below $0.1^{m}$. These errors do not show a clear dependence on the opacity as it can be seen in Table 1. This is a consequence of the ability of the method to handle systems even with very low optical depth. The errors in this case depend on the pure absorption fraction $\epsilon$ of the model. 


\section{Conclusions}

The possibility of applying to dynamical situations the closure relationship proposed by Simonneau (1979) for extended envelopes has been verified. The use of a slightly modified version of this closure relationship together with the comoving frame moment equations is a suitable method for solving the radiative transfer problem. This method takes into account velocity terms as well as scattering effects, and allows the simultaneous treatment of the optically thin and thick regions, giving much more accurate results than those obtained with the diffusion approximation. Since the closure relationship can be determined a priori, no significative complexity is added to the calculations and the computational effort is similar to that required by the diffusion approximation.

Our results show that the method provides very precise results for all the quantities in all the scenarios considered here, even at the late epochs when the envelope becomes transparent. For the same models the diffusion approximation fails in reproducing all the properties other than bolometric luminosity a month after the explosion.

Acknowledgements. We are grateful to E. Simonneau for helpful comments concerning

this paper and to R. López for information on her previous work in this subject. This work has been financed with the CICYT project ESP95-0091. 
Table 1. DEVIATIONS FROM THE STANDARD MODEL OF DIFFERENT APPROXIMATIONS ${ }^{\text {a }}$

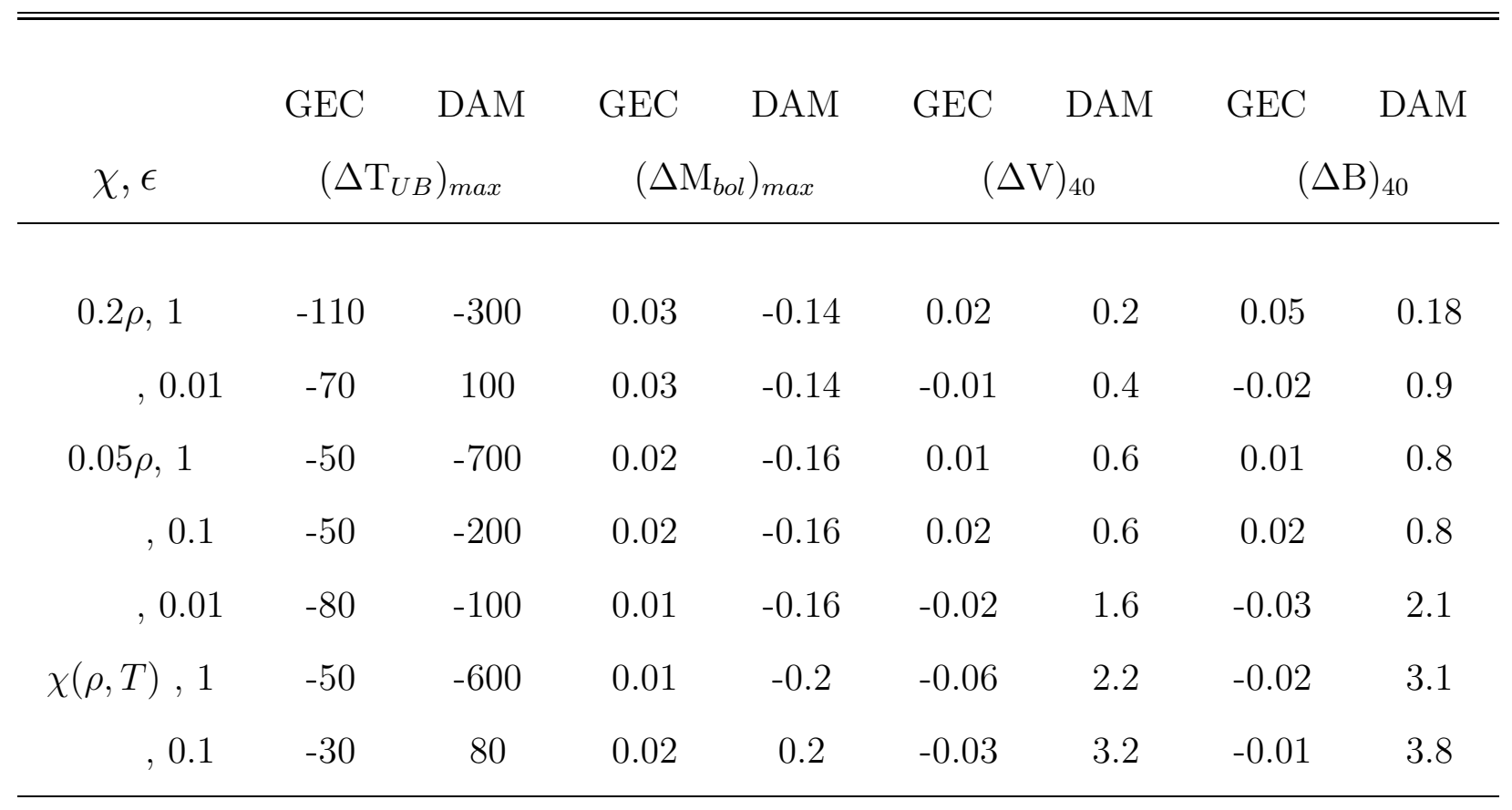

${ }^{a}$ (GEC) corresponds to simulations performed with the Simonneau's generalization of the Eddington closure and (DAM) to the simulations performed with the diffusion approximation. 


\section{REFERENCES}

Arnett, D.W. 1979, ApJ 237, 541

Arnett, D.W. 1980, ApJ 253, 785

Baron, E., Hauschildt, P.H. and Mezzacappa, A. 1996, in Proceedings of the NATO ASI on Thermonuclear Supernovae, Eds. Canal, R. Isern, J. \& Ruiz-Lapuente, P, (Kluwer) (in press)

Castor, J.I. 1972,ApJ 178, 779

Blinnikov, S.I. \& Bartunov, O.S. 1993, A\&A 273, 106

Branch, D., Buta, R., Falk, S. W., McCall, M. L., Sutherland, P. G., Uomoto, A., Wheeler, J. C. \& Wills, B. J. 1982, ApJ 252, L61

Bravo, E., Dominguez, I., Isern, J., Canal, R., Höflich, P. \& Labay, J. 1993, A\&A 269, 187

Colgate, A., Petschek, A. \& Kriese, J. 1980, ApJ 237, L81

Ensman, L. 1994, ApJ 424, 275

Höflich, P., Müller, E. \& Khokhlov, A. 1993, A\&A 268, 570

Kato, M. \& Hachisu I. 1994, A\&A 437, 802

López, R., Isern, J. \& Simonneau, E. 1987, A\&A 184, 249

Mihalas, D. 1977, in Stellar Atmospheres (San Francisco: Freeman)

Mihalas, D. and Kunasz, P. 1978, ApJ 219, 635

Mihalas, D. and Weibel-Mihalas B. 1984, in Foundations of Radiation Hydrodynamics (New York: Oxford University Press) 
Schurmann, S. R. 1983, ApJ, 267, 779

Simonneau, E. 1979, PhD. Thesis, Paris VII University

Simonneau, E., Isern, J. and López, R., 1987, A\&A 208,166

Stone, J., Mihalas, D. and Norman, M. 1992, ApJS 80, 819 
Fig. 1.- Values of $\mu_{c}$ for a SNIa 30 days after the explosion. Solid line corresponds to the static case, dashed line and dotted line correspond to the values obtained in the non static case with the comoving-frame and inertial-frame values respectively.

Fig. 2.- Bolometric light curves for the model with temperature dependent opacity and pure absorption fraction $\epsilon=1$. The light curves have been obtained with the diffusion approximation (dotted line), the proposed method (dashed line) and the variable Eddington factor method (solid line).

Fig. 3. - E rad $_{\text {rad }}$ evolution for models with $\chi=0.2 \rho$ (top) and $\epsilon=1, \chi=0.05 \rho$ and $\epsilon=1$ (bottom). Lines have the usual meaning.

Fig. 4. $-\mathrm{T}_{\text {color }}$ as a function fo the time for the models with: (a) $\chi=0.05 \rho$ and $\epsilon=1$, (b) $\chi=\chi(\rho, T)$ and $\epsilon=1$. Curve (b) is $3000 \mathrm{~K}$ below its actual position. Lines have the usual meaning.

Fig. 5. - V and B (shifted 1 magnitude) light curves. Model with $\chi=0.05 \rho$ and $\epsilon=1$. Lines have the usual meaning.

Fig. 6. - Evolution of the color index B-V for models with $\chi=0.05 \rho$. (a) $\epsilon=1$, (b) $\epsilon=0.1$. Curves of model (a) have been shifted 1 magnitude. Lines have their usual meaning. 


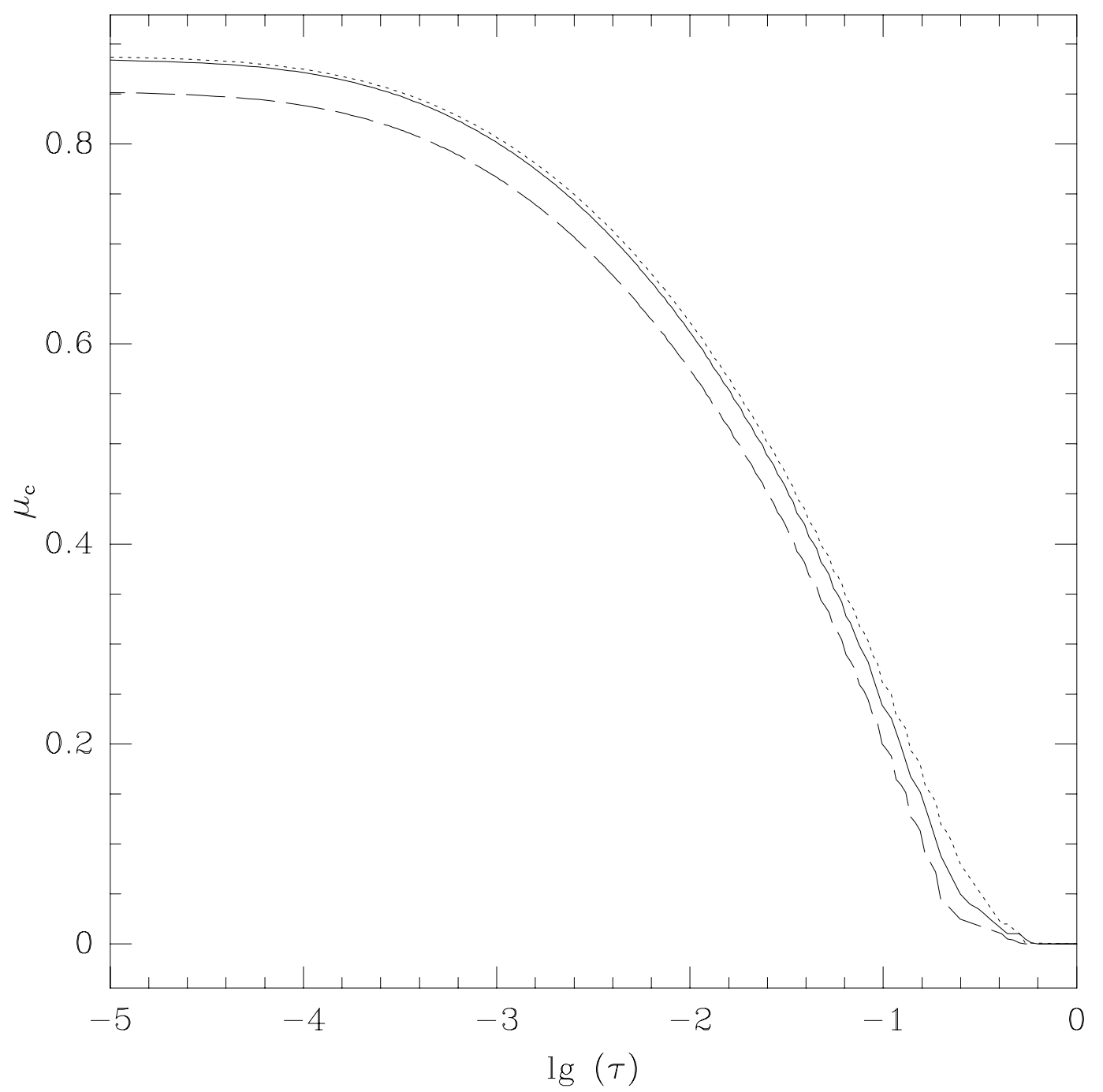




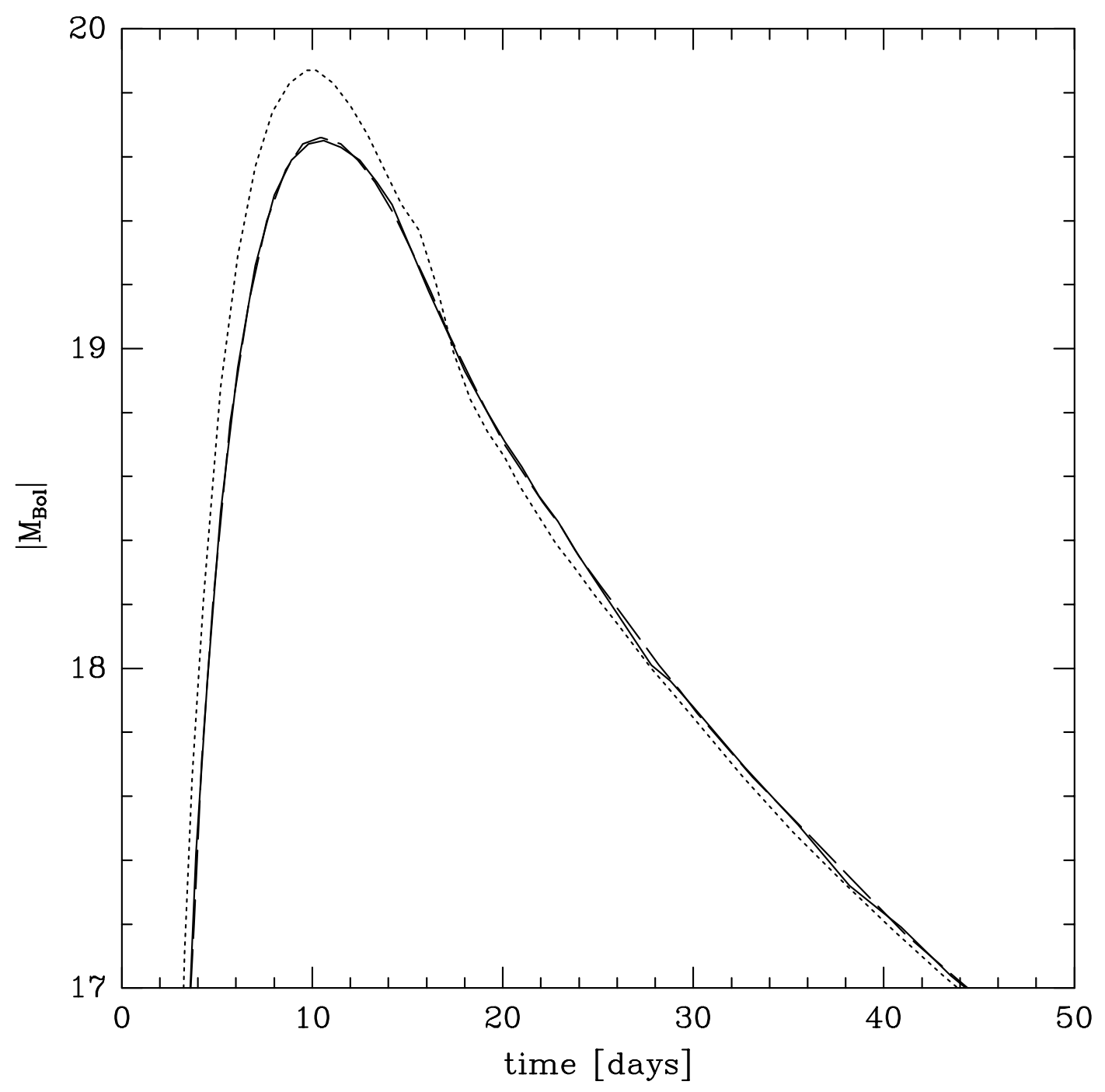




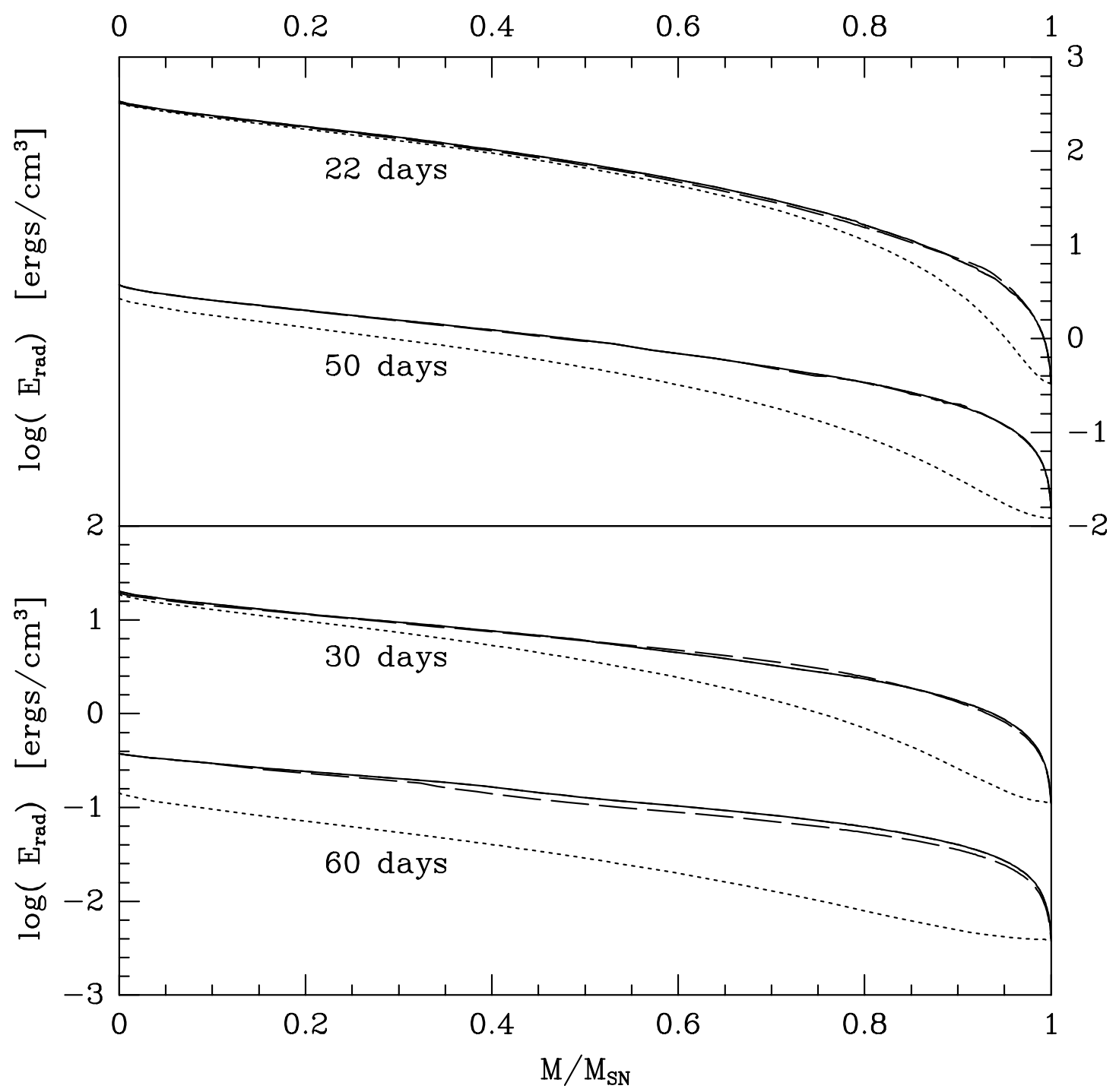




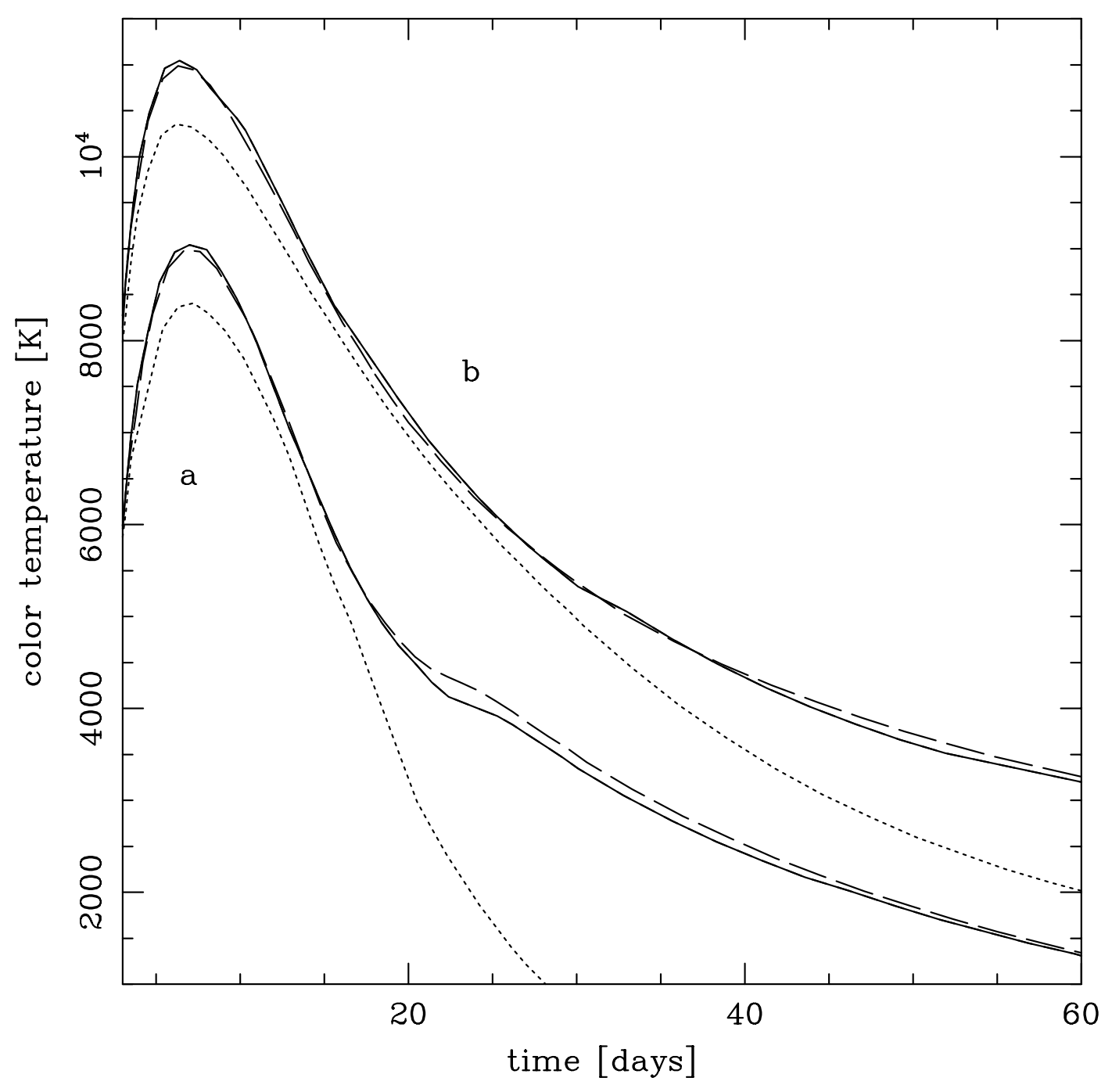




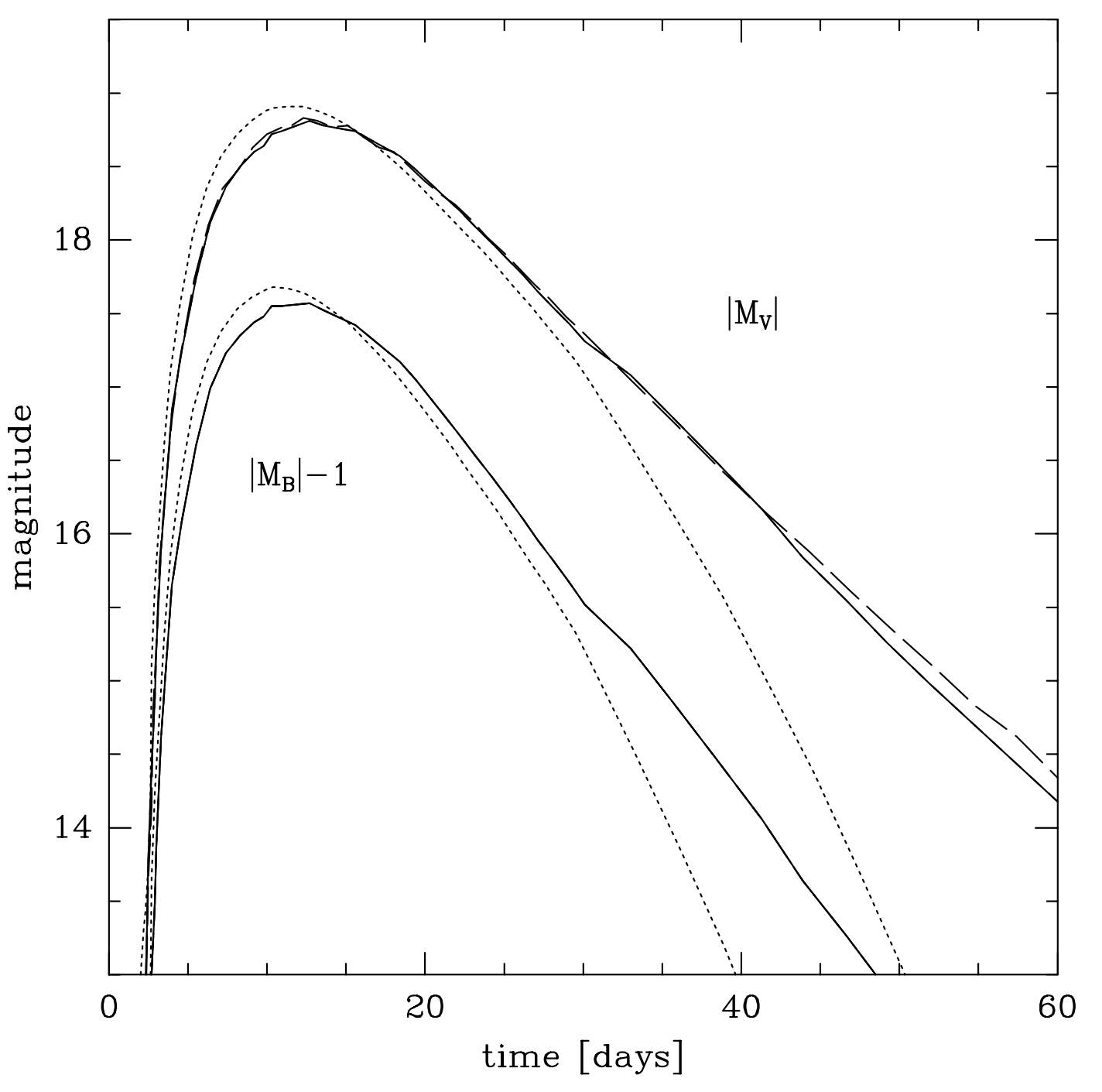




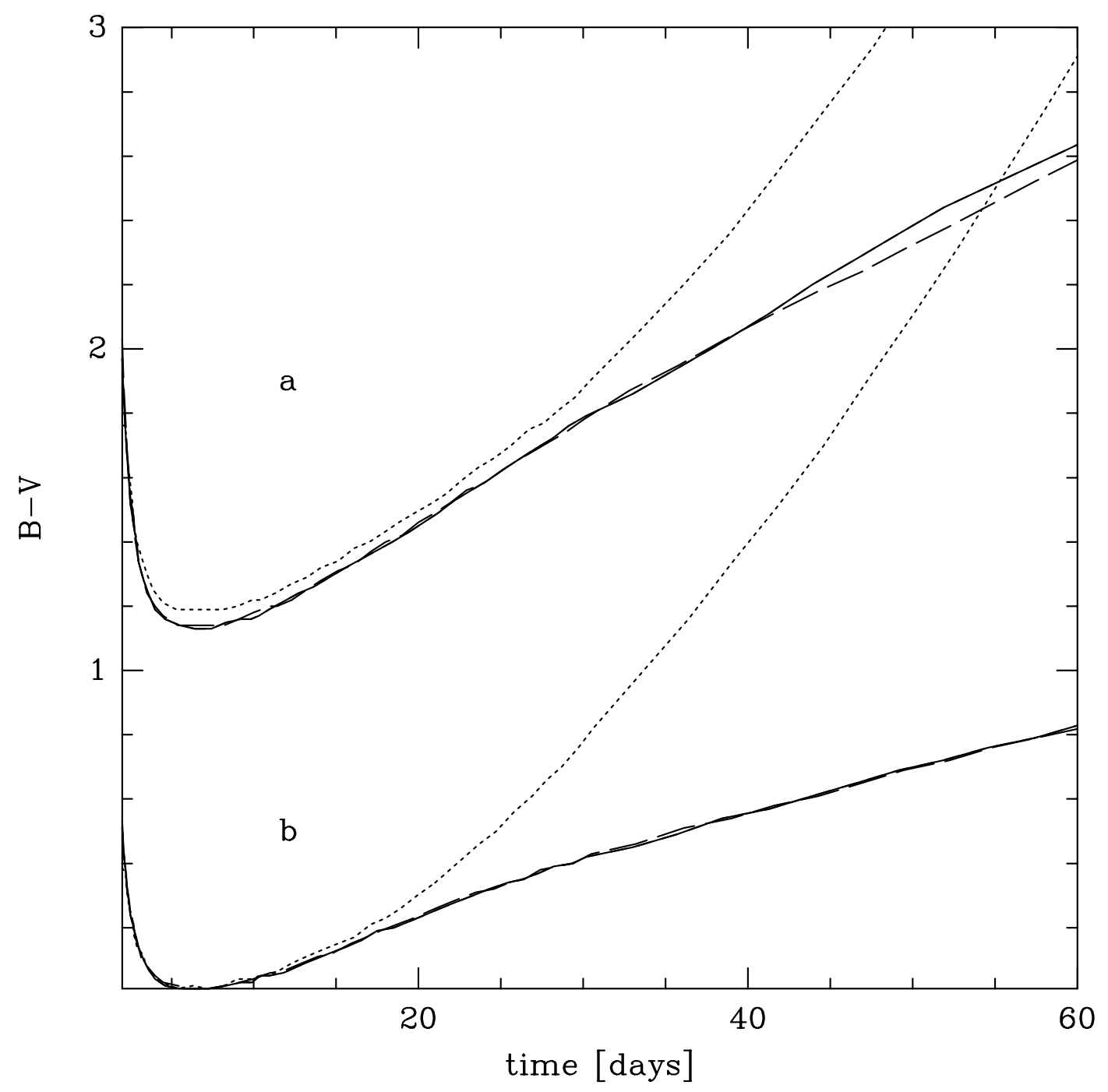

